\title{
Fecal occult blood testing for the prediction of small-bowel pathology detected by capsule endoscopy: a systematic review and meta-analysis
}

\author{
Diana E Yung a , Sanju Vijayan' ${ }^{\text {, }}$ Tomer Avnic, Sarah Douglas ${ }^{a}$, Uri Kopylov ${ }^{d}$, Anastasios Koulaouzidis ${ }^{a}$ \\ The Royal Infirmary of Edinburgh, Edinburgh, United Kingdom; The University of Edinburgh, Edinburgh, \\ United Kingdom; Rabin Medical Center, Petah-Tikva, Israel; Sheba Medical Center, Tel Hashomer, and Sackler School \\ of Medicine, Tel Aviv University, Israel
}

\section{Abstract}

Background Fecal occult blood testing (FOBT) has been suggested as a potential screening tool for small-bowel capsule endoscopy (CE). We conducted a meta-analysis of studies correlating FOBT and CE findings to examine the predictive value of positive FOBT for CE findings.

Methods PubMed and Embase search. Sensitivity, specificity and diagnostic odds ratios (DORs) were calculated.

Results Six studies were identified. Four used fecal immunochemical testing (FIT), one used FIT and guaiac FOBT, one used hemoglobin/haptoglobin complex testing $(\mathrm{Hb} / \mathrm{Hpt})$. Five of the 6 studies were suitable for statistical analysis. For all positive FOBT, sensitivity for small-bowel findings was 0.60 (95\%CI $0.50-0.69)$, specificity was 0.72 (95\%CI $0.52-0.86)$, and DOR was 3.96 (95\%CI 1.50-10.4). For the 4 studies using only FIT, sensitivity was 0.48 (95\%CI 0.36-0.61), specificity was 0.60 (95\%CI 0.42-0.76), and DOR was 1.41 (95\%CI 0.72-2.75).

Conclusions Although a number of modalities have been suggested for screening small-bowel CE referrals, none of them, including FOBT, offer a comprehensive solution. Further work is required to refine screening methods for small-bowel CE referrals.

Keywords Capsule endoscopy, fecal occult blood test, fecal immunochemical test, small bowel, meta-analysis, systematic review

Ann Gastroenterol 2017; 30 (1): 1-6

${ }^{\mathrm{a} C e n t r e ~ f o r ~ L i v e r ~ a n d ~ D i g e s t i v e ~ D i s o r d e r s, ~ T h e ~ R o y a l ~ I n f i r m a r y ~}$ of Edinburgh, Edinburgh, United Kingdom (Diana Yung, Sarah

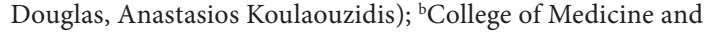
Veterinary Medicine, The University of Edinburgh, Edinburgh, United Kingdom (Sanju Vijayan); 'Department of Medicine E, Beilinson Hospital, Rabin Medical Center, Petah-Tikva, Israel (Tomer Avni); ${ }^{\mathrm{D}}$ Department of Gastroenterology, Sheba Medical Center, Tel Hashomer, and Sackler School of Medicine, Tel Aviv University, Israel (Uri Kopylov)

Conflict of Interest: Diana Yung: Previously received grant from Dr Falk Pharma/Core UK. Anastasios Koulaouzidis: Honoraria from and member of advisory board for Dr Falk Pharma UK, material support for research from Synmed UK, travel support from Almirall, Dr Falk Pharma

Correspondence to: Diana E. Yung MBChB, The Royal Infirmary of Edinburgh, Endoscopy Unit, 51 Little France Crescent, Edinburgh EH16 4SA, Edinburgh, United Kingdom, Tel.: +44 131 2421126,

e-mail: diana.e.yung@gmail.com

Received 25 October 2016; accepted 29 November 2016; published online 5 January 2017

DOI: https://doi.org/10.20524/aog.2017.0122

\section{Introduction}

Capsule endoscopy (CE) has become a prime mode of investigating the small bowel (SB), because of its high diagnostic yield (DY), minimal invasiveness and ease of use [1-3]. The DY of CE has been consistent across studies at about $60 \%$ [1]; this could be partially due to the widespread, and occasionally injudicious, use of CE [4], leading to several ultimately normal SBCE examinations [5]. Therefore, as the demand for CE increases, there is a need to develop screening pathways in order to prioritize SBCE referrals [3]. For example, a recent meta-analysis has found that fecal calprotectin has a high negative predictive value in patients undergoing $\mathrm{CE}$ for SB Crohn's disease (CD) [6].

Because of their simplicity and user-friendliness, and despite their low sensitivity, guaiac-based fecal occult blood testing (gFOBT) and fecal immunochemical testing (FIT) have found widespread use in colorectal cancer screening $[7,8]$. However, 40-60\% individuals with positive FIT (cutoff values 75 and $100 \mathrm{ng} / \mathrm{mL}$, similar to the gFOBT threshold) 
have no colorectal lesions [9], raising the possibility of SB bleeding. Overall, data on the predictive value of FOBT for SB pathology are scarce.

This study aimed to investigate the usefulness of gFOBT or FIT as a screening tool for CE, by examining the predictive value of positive gFOBT and FIT for SB lesions detected on CE.

\section{Materials and methods}

A comprehensive literature search was conducted using the PubMed and Embase databases (January 2000 to June 2016). The search was performed in July 2016. In order to capture as many articles as possible, a broad search strategy was employed by combining the terms "capsule endoscopy" and " $\mathrm{f}(\mathrm{a}) \mathrm{ecal}$ occult blood", as detailed below. The search was performed with no limitations.

A further keyword search for "capsule endoscopy" AND "fecal occult blood" was conducted in Google Scholar, turning up one more article [10], available online whilst awaiting print publication. A flowchart detailing the search and selection process is presented in Fig. 1.
For a study to be included in this meta-analysis, the following predefined inclusion criteria were applied: observational and case-control studies or series of FOB/FIT and CE in patients (both prospective and retrospective), articles published in English. Exclusion criteria were: systematic reviews and/or meta-analyses, editorials, opinion papers, studies published as abstracts only, studies involving the use of CE to visualize areas of the GI tract other than SB (e.g. colon CE).

Data extraction and quality control were performed independently by 2 reviewers (SV, DY). A third reviewer (AK), an expert in the content material, was involved if any conflict occurred. When additional data were required, primary (first and/or senior) authors of the corresponding manuscript(s) were contacted by email with relevant questions.

\section{Outcome measures}

The diagnostic accuracy (sensitivity, specificity, diagnostic odds ratio) of gFOBT/FIT in the diagnosis of SB pathology was evaluated. For the purposes of analysis, we used the diagnoses reported in each included publication, without adjusting for any structured terminology or scoring system in CE.

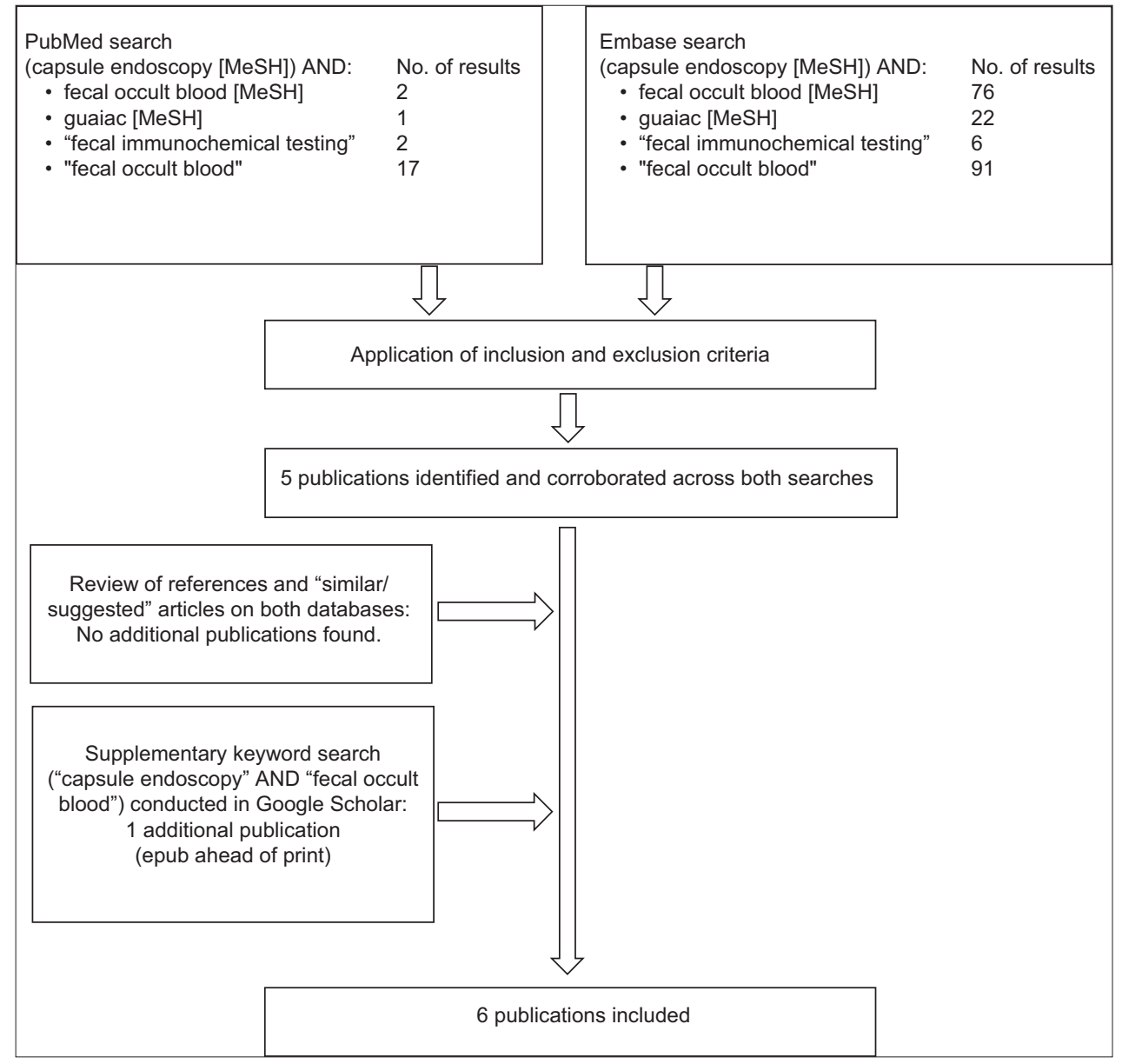

Figure 1 Flowchart for selection of studies in this meta-analysis 


\section{Statistical analysis}

We listed the number of true positive, true negative, false positive and false negative results per study, with the hemoglobin $(\mathrm{Hb})$ cutoff. We then calculated the sensitivity, specificity and diagnostic odds ratio (DOR). We used the bivariate model for data summary. Parameter estimates from the model were used to obtain hierarchical summary receiver operating curves, with $95 \%$ confidence intervals (CI), and a $95 \%$ prediction region defining the sensitivity and specificity values within which we could expect the results of a future study to lie. Only direct test comparisons were performed. Because of the statistical heterogeneity, the DerSimonian-Laird random effects model was applied. The methodological quality of the included studies was evaluated using the quality assessment of diagnostic accuracy studies (QUADAS) 2 scale [11]. Analyses were conducted using the STATA 12 (StataCorp, College Station, Texas, USA) and RevMan 5.3 (Cochrane Corporation, London, UK) software packages.

\section{Results}

Six studies [5,10,12-15] were included for review, with a total of 607 patients (380 male/227 female). Four [10,12-14] used FIT (Eiken Chem, Tokyo, Japan), with an Hb cutoff of $100 \mathrm{ng} / \mathrm{mL}$; one [5] used both FIT (Onsight, Laboratory diagnostics; cutoff $100 \mathrm{ng} / \mathrm{mL}$ ) and gFOBT (Stanbio, Laboratory diagnostics); and one [15] used the Hb/haptoglobin (Hpt) complex test (Eiken Chem, Tokyo, Japan) with a cutoff of $0.1 \mathrm{ng} / \mathrm{mL}$. All studies were published between 2011 and 2016. Four [10,12,13,15] were carried out in Japan, one [5] in Australia and one [14] in Israel; all were single-center studies. Moreover, all but one [13] of the studies were prospective. All studies used capsule endoscopes from Medtronic: one study [5] used the $\mathrm{M} 2 \mathrm{~A}^{\circledR}$ capsule and all others used various versions of PillCam ${ }^{\circledR}$, i.e. SB/SB2/SB3. The number of $\mathrm{CE}$ readers ranged from 1 to 3 . The time between FOBT and CE ranged from 3 days to 4 months; in most studies the time lag was in the range of 1-2 weeks. All studies were designed so that the higher/highest FOBT result was considered; most studies used 1 of 2 samples, whilst one study used 1 of 3 [14]. The majority of the indications for CE referral were obscure gastrointestinal (GI) bleeding (both occult and overt), following negative bidirectional GI endoscopy. Table 1 details the included studies.

Overall, 5/6 studies were suitable for statistical analysis; one study was excluded from the analysis [12] as it included only patients with positive FIT and was therefore unsuitable for this meta-analysis. When all positive FOBT results regardless of type of test were analyzed (Fig. 2), the sensitivity for SB findings was 0.60 (95\%CI $0.50-0.69)$, specificity was 0.72 (95\%CI 0.52 $0.86)$, and the DOR of any positive FOBT was 3.96 (95\%CI 1.50-10.4).

Four studies $[5,10,13,14]$ dealing only with FIT were analyzed (Fig. 3). The sensitivity of FIT for SB findings on CE was 0.48 (95\%CI $0.36-0.61)$, specificity was 0.60 (95\%CI 0.42 $0.76)$, and the DOR of positive FIT was 1.41 (95\%CI 0.72-2.75).

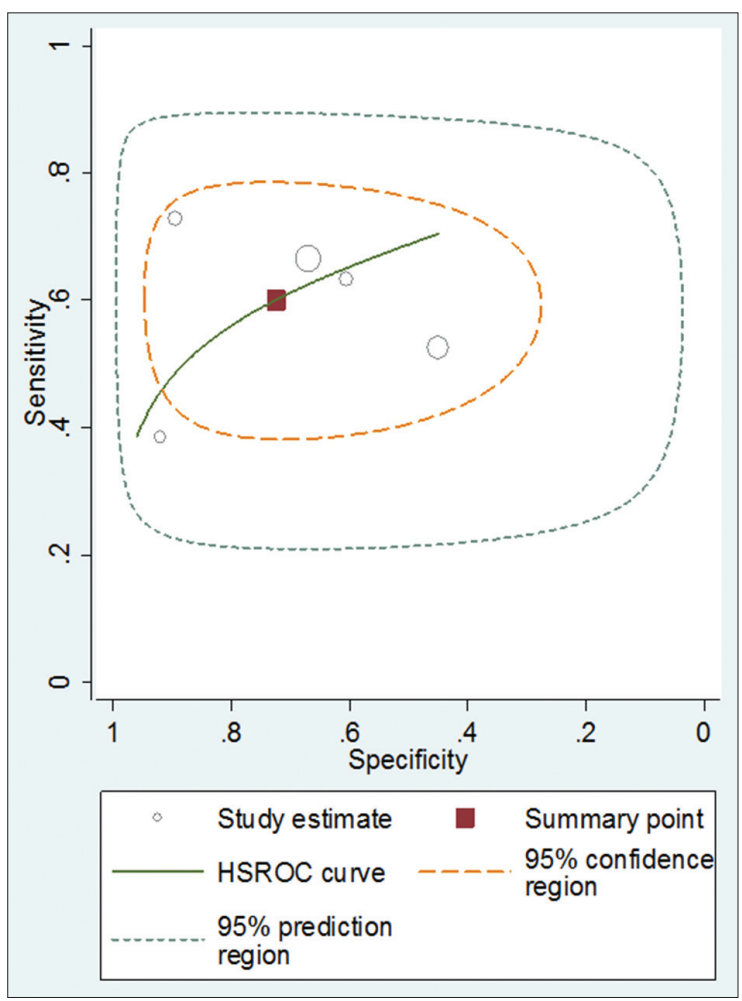

Figure 2 Hierarchical summary receiver operating characteristic (HSROC) curve of all fecal occult blood tests for prediction of smallbowel pathology on capsule endoscopy

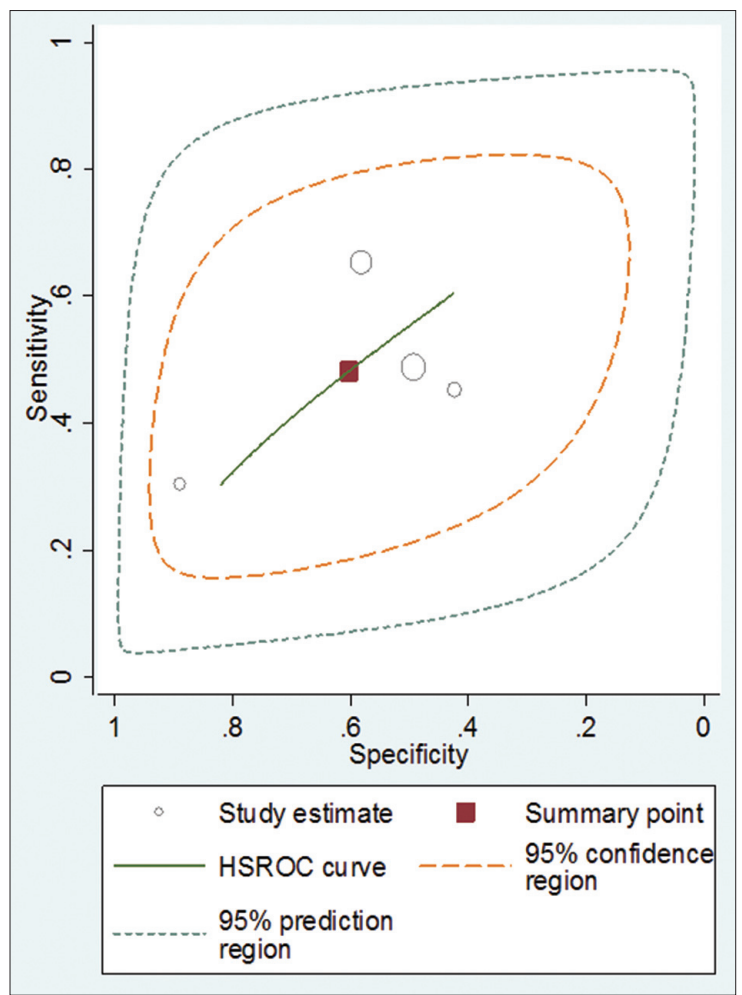

Figure 3 Hierarchical summary receiver operating characteristic (HSROC) curve of all fecal immunochemical tests for prediction of small-bowel pathology on capsule endoscopy 


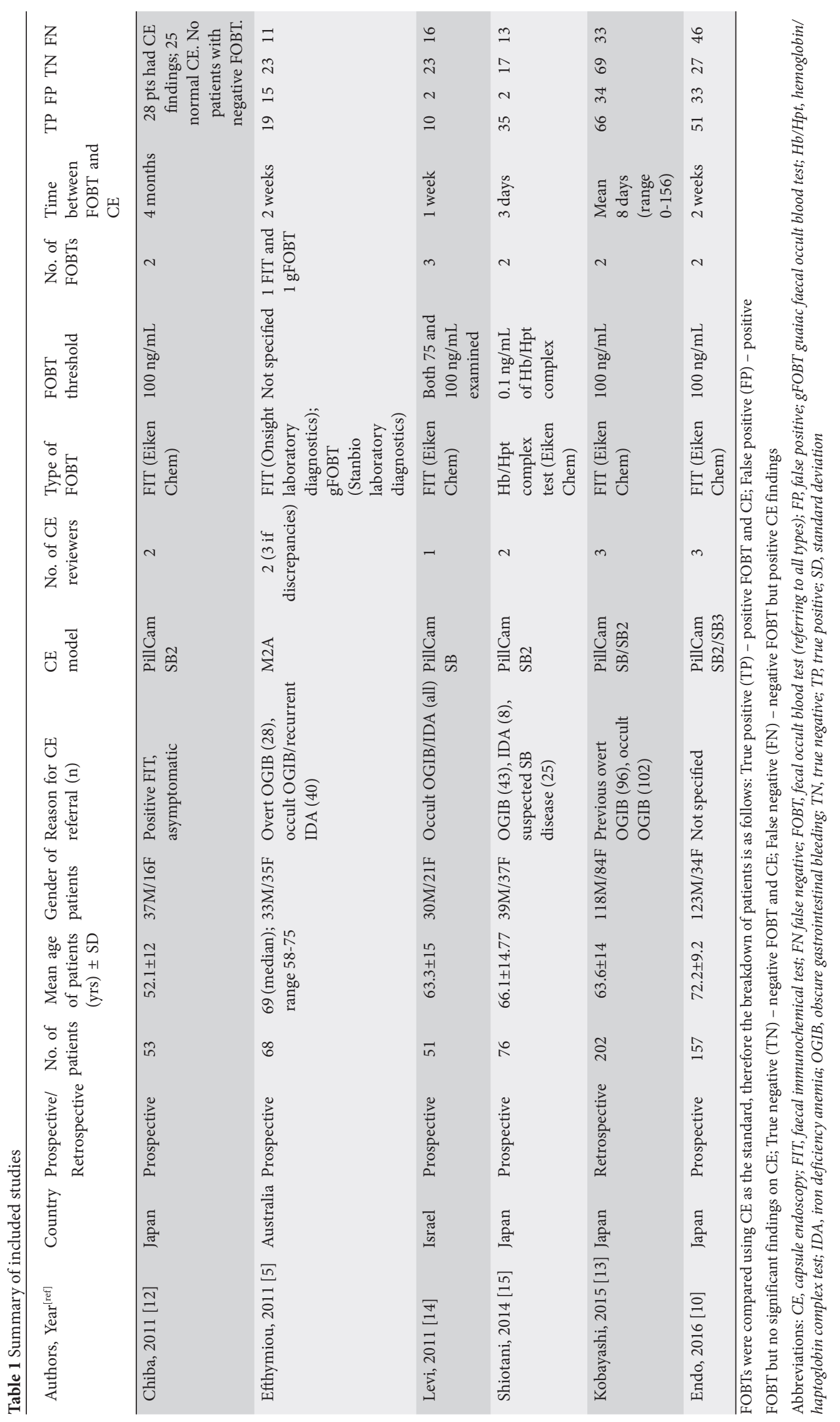




\section{Subgroup analyses}

When we examined studies where only FIT produced by Eiken Chem was used [10,13-15], we obtained a sensitivity of 0.59 (95\%CI $0.47-0.70$ ), a specificity of 0.76 (95\%CI 0.51 0.91 ) and a DOR of 4.51 (95\%CI 1.36-15.7). When only prospective studies were included $[5,10,14,15]$, the sensitivity was 0.58 (95\%CI 0.46-0.69), specificity was 0.75 (95\%CI 0.49 $0.90)$, and DOR was 4.10 (95\%CI 1.14-14.8).

\section{QUADAS-2}

The included studies had a low risk of bias, except for one study [12] that examined FOBT positive patients only. The results of the quality analysis are summarized in Table 2.

\section{Discussion}

FOBT detects the presence of traces of blood in stool [7]. Conventional gFOBT is based on guaiac, which detects pseudoperoxidase activity and is therefore not specific for human blood [8]. Conversely, FIT specifically detects human blood by antibody reaction to human globin. Moreover, it is thought to be more specific for distal GI blood loss and also has the advantage of providing quantitative results [16,17]. $\mathrm{Hb} / \mathrm{Hpt}$ complex testing is a variant of FIT that uses an immunoradiometric assay for the $\mathrm{Hb} / \mathrm{Hpt}$ complex. Hpt forms a soluble complex with $\mathrm{Hb}$, which remains stable even following incubation with gastric juice and fecal extracts [15].

Our results show the low sensitivity and specificity of FOBT, and specifically of FIT, for SB findings on CE. Overall, the attempted use of FIT as a selection tool for SBCE showed a sensitivity of 0.48 and specificity of 0.60 ; i.e. neither positive nor negative FIT results are good predictors of SB findings on CE. However, when all positive FOBT methods (i.e. gFOBT, FIT

Table 2 Quality assessment of diagnostic accuracy studies (QUADAS) 2 results for the included studies, "+" denotes low risk of bias, “-” high risk of bias, and "?" unclear risk.

\begin{tabular}{|c|c|c|c|c|c|c|c|}
\hline Author, year [ref] & 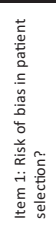 & 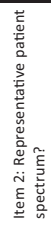 & 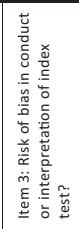 & 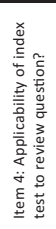 & 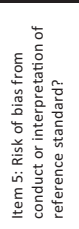 & 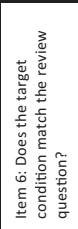 & 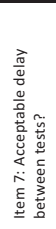 \\
\hline Chiba, 2011 [12] & & & 1 & & 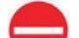 & 4 & 1 \\
\hline Efthymiou, 2011 [5] & & & & & & & \\
\hline Levi, 2011 [14] & & & 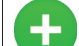 & & & & \\
\hline Shiotani, 2014 [15] & & & & & & & \\
\hline Kobayashi, $2015[13$ & & & & & & & \\
\hline Endo, 2016 [10] & & & & & & & \\
\hline
\end{tabular}

and $\mathrm{Hb} / \mathrm{Hpt}$ testing) were combined, although the sensitivity remained low (0.60), specificity increased to 0.72 and the DOR was also improved. Nevertheless, none of the aforementioned meta-analysis parameters are sufficiently significant to support the use of FOBT as a screening tool for SBCE.

$\mathrm{CE}$ is currently the third-line test following negative bidirectional GI endoscopy. There is good supporting evidence for its use in obscure GI bleeding [18]. However, the DY of CE (although superior to other modalities [3]), remains less than ideal. This could be due to the inherent limitations of commercially available capsules [19], but may also be due to pressures on $\mathrm{CE}$ services nationwide and/or inappropriate referrals being made [4]. CE remains a labor-intensive investigative modality; therefore, despite increasing use and demand, there is the need to rationalize SBCE referrals $[1,4]$.

There are currently no validated selection tools for CE referral, while the most recent European Society of Gastrointestinal Endoscopy guidelines state that there is insufficient evidence concerning the use of FOBT as a CE screening tool [1]. Referring to our previous work on young patients with iron deficiency anemia [20], the available data on the use of CE in specific subgroups of patients with SB bleeding [21] are minimal and present future research opportunities. A recent meta-analysis from our group examined the use of fecal calprotectin as a selection tool for CE, finding that it displayed good sensitivity and specificity at a cutoff of $50 \mu \mathrm{g} / \mathrm{g}$ [6].

FOBT is currently in nationwide use for colorectal cancer screening. There is a good amount of evidence regarding the usefulness of FIT in colon cancer, especially as it is more specific to lower GI tract blood loss $[7,17,22]$. Current national threshold levels for FIT are $100 \mathrm{ng} / \mathrm{mL}$ and there was only one study included here which investigated a lower threshold of $75 \mathrm{ng} / \mathrm{mL}$. Although in a study by Levi et al [14] both thresholds corresponded well with significant SB lesions, our meta-analysis has shown that FIT has not performed well overall.

Limitations of this meta-analysis include the small number of included articles, emphasizing the paucity of literature on this topic. There was also a lack of standardization between the included studies in lesion classification. Therefore, the definition of a positive SBCE examination may have differed between studies, with some studies reporting all SB findings while others may have considered only clinically significant findings. Furthermore, what is clinically significant or positive may vary with the indication for SBCE and with the SBCE reader's opinion and/or expertise. This limitation is highlighted by an American Gastroenterological Association technical review on occult and obscure GI bleeding, which suggested that the criteria used to determine the source of positive FOBT or iron deficiency anemia are not standardized, as GI mucosal lesions are rarely actively bleeding or showing stigmata of recent bleeding at the time of endoscopy [18].

In conclusion, although a number of modalities, including FOBT, have been suggested for screening SBCE referrals, this meta-analysis shows that FOBT does not offer a comprehensive solution. Further work is required to refine screening methods, such as combining other fecal or serum markers, for the selection of patients for SBCE. This would help rationalize the use of valuable resources. 


\section{Summary Box}

\section{What is already known:}

- Small-bowel capsule endoscopy (SBCE) is invaluable for minimally-invasive SB examination

- The accuracy of SBCE could be improved by more judicious patient selection

- Selection pathways using easily obtained and measured biomarkers are desirable to rationalize the use of scarce resources

\section{What the new findings are:}

- Fecal occult blood testing (FOBT) has limited predictive value for SB findings overall

- The overall specificity (72\%) of FOBT for SB findings is superior to its sensitivity (60\%)

- Other fecal or serum markers could be used in conjunction with FOBT to improve the selection of patients prior to SBCE

\section{References}

1. Pennazio M, Spada C, Eliakim R, et al. Small-bowel capsule endoscopy and device-assisted enteroscopy for diagnosis and treatment of small-bowel disorders: European Society of Gastrointestinal Endoscopy (ESGE) Clinical Guideline. Endoscopy 2015;47:352-376.

2. Wang A, Banerjee S, Barth BA, et al; ASGE Technology Committee. Wireless capsule endoscopy. Gastrointest Endosc 2013;78:805-815.

3. Koulaouzidis A, Rondonotti E, Karargyris A. Small-bowel capsule endoscopy: a ten-point contemporary review. World J Gastroenterol 2013;19:3726-3746.

4. Gerson LB. Use and misuse of small bowel video capsule endoscopy in clinical practice. Clin Gastroenterol Hepatol 2013;11:1224-1231.

5. Efthymiou M, Allen PB, Jayasekera C, Taylor PV, Taylor AC. Value of fecal occult blood test as a screening test before capsule endoscopy. Eur J Gastroenterol Hepatol 2011;23:690-694.

6. Kopylov U, Yung DE, Engel T, et al. Fecal calprotectin for the prediction of small-bowel Crohn's disease by capsule endoscopy: a systematic review and meta-analysis. Eur J Gastroenterol Hepatol 2016;28:1137-1144.

7. Zorzi M, Fedeli U, Schievano E, et al. Impact on colorectal cancer mortality of screening programmes based on the faecal immunochemical test. Gut 2015;64:784-790.

8. Hewitson P, Glasziou P, Watson E, Towler B, Irwig L. Cochrane systematic review of colorectal cancer screening using the fecal occult blood test (hemoccult): an update. Am J Gastroenterol 2008; 103:1541-1549.

9. Jun J, Jae P, Cheon H. Small bowel evaluation in asymptomatic fecal immunochemical test-positive patients with a negative colonoscopy: is it necessary? Dig Dis Sci 2011;56:2773-2775.

10. Endo H, Kato T, Sakai E, et al. Is a fecal occult blood test a useful tool for judging whether to perform capsule endoscopy in low-dose aspirin users with negative colonoscopy and esophagogastroduodenoscopy? J Gastroenterol 2016 Apr 19. [Epub ahead of print]

11. Whiting PF, Rutjes AWS, Westwood ME, et al. Quadas-2: a revised tool for the quality assessment of diagnostic accuracy studies. Ann Int Med 2011;155:529-536.

12. Chiba H, Sekiguchi M, Ito T, Tsuji Y, Ohata K. Is it worthwhile to perform capsule endoscopy for asymptomatic patients with positive immunochemical faecal occult blood test? Dig Dis Sci 2011;56:3459-3462.

13. Kobayashi Y, Watabe H, Yamada A, et al. Impact of fecal occult blood on obscure gastrointestinal bleeding: observational study. World J Gastroenterol 2015;21:326-332.

14. Levi Z, Gal E, Vilkin A, et al. Fecal immunochemical test and small bowel lesions detected on capsule endoscopy: results of a prospective study in patients with obscure occult gastrointestinal bleeding. Eur J Gastroenterol Hepatol 2011;23:1024-1028.

15. Shiotani A, Tarumi K, Honda K, et al. Application of fecal hemoglobin-haptoglobin complex testing for small bowel lesions. Scand J Gastroenterol 2014;49:539-544.

16. Hol L, van Leerdam ME, van Ballegooijen M, et al. Screening for colorectal cancer: randomised trial comparing guaiac-based and immunochemical faecal occult blood testing and flexible sigmoidoscopy. Gut 2010;59:62-68.

17. Brenner H, Tao S. Superior diagnostic performance of faecal immunochemical tests for haemoglobin in a head-to-head comparison with guaiac based faecal occult blood test among 2235 participants of screening colonoscopy. Eur J Cancer 2013;49:30493054 .

18. Zuckerman GR, Prakash C, Askin MP, Lewis BS. AGA technical review on the evaluation and management of occult and obscure gastrointestinal bleeding. Gastroenterology 2000;118:201-221.

19. Rondonotti E, Herrerias JM, Pennazio M, Caunedo A, MascarenhasSaraiva M, de Franchis R. Complications, limitations, and failures of capsule endoscopy: a review of 733 cases. Gastrointest Endosc 2005;62:712-716.

20. Koulaouzidis A, Yung DE, Lam JH, Smirnidis A, Douglas S, Plevris JN. The use of small-bowel capsule endoscopy in irondeficiency anemia alone; be aware of the young anemic patient. Scand J Gastroenterol 2012;47:1094-1100.

21. Goddard AF, James MW, McIntyre AS, Scott BB; British Society of Gastroenterology. Guidelines for the management of iron deficiency anaemia. Gut 2011;60:1309-1316.

22. Vart G, Banzi R, Minozzi S. Comparing participation rates between immunochemical and guaiac faecal occult blood tests: a systematic review and meta-analysis. Prev Med 2012;55:87-92. 